\title{
Presentación. \\ Transiciones educativas y desigualdades sociales: una perspectiva sociológica
}

\author{
Aina Tarabini \\ Universitat Autònoma de Barcelona \\ aina.tarabini@uab.cat
}

\section{Resumen}

Esta presentación tiene como objetivo contextualizar y presentar los trabajos incluidos en el número monográfico de la revista Papers titulado «Transiciones educativas y desigualdades sociales: una perspectiva sociológica». Asimismo, pretende explicar la importancia de este tema para la investigación y la reflexión sociológica. Las transiciones educativas tienen una relevancia clave para mostrar las dinámicas de desigualdad social, especialmente en las etapas posobligatorias. Asimismo, los momentos de transición educativa son especialmente indicados para el estudio de las interacciones entre estructura y agencia, tan arraigados dentro de la perspectiva sociológica en general y de la sociología de la educación y de la juventud en particular. Finalmente, la investigación especializada ha enfatizado de forma creciente la importancia de estudiar las elecciones y transiciones de los y las jóvenes para entender el proceso de configuración de sus identidades. Por todo ello, el análisis de las transiciones educativas es un objeto de estudio especialmente pertinente para la sociología. Las contribuciones del monográfico, de forma individual y colectiva, ayudan a identificar una gran diversidad de factores sistémicos, institucionales y subjetivos que suponen un aporte clave para el estudio de las transiciones educativas de los y las jóvenes desde una perspectiva sociológica.

Palabras clave: transiciones educativas; desigualdad social; sociología 
Abstract. Presentation. Educational transitions and social inequalities: a sociological perspective

This presentation aims to contextualize and introduce the articles included in the Special Issue of Papers «Educational transitions and social inequalities: a sociological perspective». It also aims to explain the importance of this topic for a sociological research and reflection. Educational transitions have a key relevance to explain the dynamics of social inequality, especially in the post-compulsory stages. Likewise, the moments of educational transition are especially indicated for the study of the interactions between structure and agency, deeply embedded within the sociological perspective in general and within the sociology of education and youth, in particular. Finally, specialized research has increasingly emphasized the importance of studying young people's choices and transitions in order to understand the process of their identity formation. For all these reasons, the study of the educational transitions is an object of study especially relevant for sociology. The contributions of the Special Issue individually and collectively contribute to identifying a great diversity of factors at the systemic, institutional and subjective levels representing a key contribution to the study of young people' educational transitions from a sociological perspective.

Keywords: educational transitions; social inequalities; sociology

Los momentos de transición educativa tienen una importancia clave para explicar las dinámicas de desigualdad social, especialmente en las etapas posobligatorias. En el sistema educativo español, obligatorio y formalmente comprensivo hasta los 16 años de edad, es a partir de la educación posobligatoria cuando, por primera vez, se abren opciones de elección «reales» en las trayectorias educativas de los y las jóvenes, tanto respecto a continuar estudiando o no como con relación a diferentes itinerarios formativos, fundamentalmente el académico (Bachillerato) y el profesional (Ciclos Formativo de Grado Medio). Tanto en el ámbito nacional como en el internacional, numerosos estudios han señalado la importancia de entender estos procesos de transición como espacios clave de selectividad social (Bernardi y Requena, 2010; Blossfeld y Shavit, 2011; Tarabini e Ingram, 2018). Es por ello que el estudio de las transiciones educativas y su papel en la (re)producción de desigualdades sociales es un objeto especialmente pertinente para la sociología.

Asimismo, los momentos de transición educativa son especialmente indicados para el estudio de las interacciones entre estructura y agencia, tan arraigados dentro de la perspectiva sociológica en general y de la sociología de la educación y de la juventud en particular. De hecho, el debate estructura-agencia ha sido un tema central en la investigación sobre trayectorias juveniles desde la publicación, a principios de los años noventa del siglo pasado, de las teorías de Giddens (1991) y Beck (1992). Los conceptos de likestyle choices (Beck, 1992) o de reflexive life planning (Giddens, 1991) han influenciado con fuerza las investigaciones desarrolladas en este campo. Bajo estas aproximaciones, numerosos autores afirman que hoy en día las trayectorias educativas de las personas jóvenes son mucho menos lineales, predecibles y anticipables de lo que eran bajo los auspicios de la modernidad y la industrialización (Bois-Reymond, 
1998; Furlong y Cartmel, 1997). Como señalan Walther et al. (2015), analizar cómo se producen los procesos de decisión entre los y las jóvenes es hoy en día fundamental para entender la complejidad de sus trayectorias educativas $y$, por ende, de las potenciales desigualdades y oportunidades asociadas a las mismas.

En este escenario, la investigación especializada ha enfatizado recientemente el estudio de la relación entre elecciones educativas, transiciones y configuración de las identidades juveniles. De hecho, los y las jóvenes atribuyen sentido a sus experiencias y a sus elecciones educativas como parte de un proceso complejo de formación de su propia identidad (Hattam y Smyth, 2003). Una identidad que siempre es múltiple, modificable y, sobre todo, relacional. Es decir, construida en relación con los otros y en contextos específicos. Como afirma Hall (1996), las identidades tienen más que ver con un proceso que con un estado. Y lo mismo afirman Cuconato y Walther (2015) para el caso específico de las transiciones educativas. Las transiciones, en palabras de los autores, son, se hacen en relación con los otros; forman parte de un proceso complejo de negociación donde los «otros significativos» tienen un papel fundamental.

Las escuelas, en particular, tienen un rol clave en la formación de la identidad y, por ende, de las decisiones y transiciones educativas de los jóvenes. Las normas, las prácticas y las expectativas escolares generan materiales simbólicos relevantes sobre los cuales los y las estudiantes construyen sus experiencias y se definen a sí mismos (Perry, 2002, en Reay, 2010: 4). Son, en palabras de Van Zanten y Legravre (2014), espacios institucionales centrales para entender la configuración de experiencias, decisiones y trayectorias educativas de los y las jóvenes.

La relación entre transiciones educativas y los tres temas de análisis señalados en las líneas anteriores - a saber: desigualdad social, estructura-agencia y proceso de formación de identidades - hace de éste un tema de especial interés sociológico que, como tal, ha sido todavía poco desarrollado en el contexto español. El objetivo del monográfico que se presenta es precisamente contribuir a llenar este vacío con un conjunto de artículos que abordan las transiciones a la educación secundaria posobligatoria en España desde una perspectiva sociológica. En particular, el monográfico incluye cinco artículos que siguen a esta introducción:

El primer artículo del monográfico, a cargo de Miriam Prieto y Javier Rujas, analiza el peso de los factores políticos e institucionales para entender las transiciones a la educación secundaria posobligatoria en la ciudad Madrid. A partir del análisis de entrevistas a agentes políticos, educativos y empresariales, de la normativa y de datos de fuentes secundarias, el artículo analiza los condicionantes del acceso y la elección tanto del Bachillerato y como de la Formación Profesional de Grado Medio, y muestra sus implicaciones en clave de desigualdad social. Como argumentan sus autores, la configuración de la oferta posobligatoria y el diseño institucional de la misma contribuyen a consolidar un prestigio extremadamente desigual entre ambas vías formativas y limitan las posibilidades de elección de los grupos en situación de mayor vulnerabilidad social.

El segundo artículo, a cargo de Aina Tarabini, Alba Castejón y Marta Curran, pone el foco en el papel de los centros educativos y, en particular, de 
los y las docentes para entender la generación de diferentes oportunidades de transición a la educación posobligatoria. A partir de entrevistas en profundidad a docentes de Educación Secundaria Obligatoria, Bachillerato y Formación Profesional (FP) de diversos institutos de la ciudad de Barcelona, las autoras muestran el rol central que ejercen las expectativas docentes para entender las pautas de desigualdad social asociadas con las transiciones educativas de los y las jóvenes. En particular, el artículo muestra una concepción ampliamente dicotómica del alumnado de Bachillerato y de FP, en términos de "capacidades», "habilidades» $\mathrm{y}$ "carácter», que contribuye a legitimar un sistema de educación posobligatoria altamente segmentado y desigual.

Los artículos tres, cuatro y cinco se focalizan específicamente en el punto de vista de los y las jóvenes, y los tres se centran en la ciudad de Barcelona. En el primer caso, Maribel García y Albert Sánchez exploran la heterogeneidad de perfiles de alumnado y de itinerarios educativos que conducen al abandono escolar una vez concluidos los estudios obligatorios. A partir de un análisis longitudinal, los autores identifican los factores generadores de vulnerabilidad y de riesgo de abandono escolar, así como los momentos críticos de ruptura escolar que se expresan durante las trayectorias educativas de los y las jóvenes. La experiencia escolar subjetiva, los bajos resultados escolares y el auto-concepto escolar se identifican como factores centrales para entender estos procesos y, de acuerdo con los mismos, se proponen diversas estrategias de intervención que permitan ampliar las oportunidades educativas de todo el alumnado, especialmente de aquel más vulnerable tanto en el ámbito social como en el escolar.

A continuación, el artículo elaborado por Judith Jacovkis, Alejandro Montes y Martí Manzano analiza la configuración de las expectativas y aspiraciones educativas de jóvenes que acaban de transitar hacia la educación secundaria posobligatoria y que, por tanto, están cursando el primer año de Bachillerato o FP. A partir de una propuesta teórica basada en la «agencia delimitada», los autores analizan el impacto de variables de estructura social y de sistema educativo en la articulación de dichas expectativas y aspiraciones. Los resultados del análisis ponen de manifiesto, por una parte, el impacto que juegan la clase social, la generación migratoria y el género del alumnado en la construcción de sus escenarios y oportunidades de futuro. Por otra, muestran el efecto crucial que ejerce la separación del sistema educativo en dos itinerarios formativos (Bachillerato y FP) sobre las expectativas y las aspiraciones de los jóvenes, incluso por encima del que tienen el resto de variables estructurales.

El último artículo del monográfico, elaborado por Rafael Merino, José Saturnino Martínez y Ona Valls analiza, basándose en la perspectiva de los «efectos secundarios», las expectativas educativas de los y las jóvenes respecto a qué camino escoger después de la educación obligatoria y, específicamente, entre la opción académica y la profesional. Asimismo, explora las motivaciones de las personas jóvenes que han escogido la opción de FP. Los resultados del análisis muestran, por una parte, que el capital cultural familiar y los resultados académicos son dos factores centrales para entender las expectativas de cursar Bachillerato o FP. Por otra, ponen de manifiesto la diversidad de motivaciones 
que existen entre los y las jóvenes para escoger FP, tanto respecto a tipologías de motivación como al grado de autonomía de las mismas.

En conjunto, el monográfico contribuye a identificar una gran diversidad de factores sistémicos, institucionales y subjetivos que suponen un aporte clave para el estudio de las transiciones educativas de los y las jóvenes desde una perspectiva sociológica.

\section{Referencias bibliográficas}

BECK, Ulrich (1992). Risk Society: Towards a New Modernity. Londres: Sage.

Bernardi, Fabrizio; Requena, Miguel (2010). «Desigualdad y puntos de inflexión educativos: el caso de la educación post-obligatoria en España». Revista de Educacion, número extraordinario, 93-118.

Blossfeld, Hans-Peter; Shavit, Yossi (2011). «Persisting Barriers: Changes in Educational Opportunities in Thirteen Countries». En: Arum, Richard; Beattie, Irenee R.; FORD, Karly (eds.). The structure of Schooling. Readings in Sociology of Education. Londres: Sage.

Du Bois-Reymond, Manuela (1998). «I Don't Want to Commit Myself Yet: Young People's Life Concepts». Journal of Youth Studies, 1 (1), 63-79. $<$ https://doi.org/10.1080/13676261.1998.10592995>.

Cuconato, Morena; Walther, Andreas (2015). «"Doing transitions" in education». International Journal of Qualitative Studies in Education, 28 (3), 283-296. $<$ https://doi.org/10.1080/09518398.2014.987851>.

Giddens, Anthony (1991). Modernity and Self-Identity: Self and Society in the Late Modern Age. Cambridge: Polity Press.

Furlong, Andy; Cartmel, Fred (1997). Young People and Social Change: Individualisation and Risk in Late Modernity. Buckingham: Open University.

Hall, Stuart (1996). «Introduction: Who needs “identity?”». En: Hall, Stuart; GaY, Paul du (eds). Questions of Cultural Identity. Londres: Sage.

Hattam, Robert; Smyth, John (2003). «Not Everyone Has a Perfect Life: becoming somebody without school». Pedagogy, Culture \& Society, 11 (3). $<$ https://doi.org/10.1080/14681360300200180>.

Reay, Diane (2010). «Identity Making in Schools and Classrooms». En: Wetherell, Margaret; MohanTy, Chandra T. (eds.). The SAGE handbook of identities. Londres: Sage Publications.

Tarabini, Aina; Ingram, Nicola (eds.). (2018). Educational Choices, Transitions and Aspirations in Europe: Systemic, Institutional and Subjective Challenges. Londres: Routledge.

Van Zanten, Agnès; Legavre, Amélia (20I4). «Engineering Access to higher education through higher education fairs». En: Goastellec, Gaële; Picard, France (eds.). The Roles of Higher Education and Research in the Fabric of Societies. Leuven: Sense Publishers.

Walther, Andreas; Warth, Annegret; Ule, Mirjana; Bois-Reymond, Manuela du (2015). " "Me, my education and I": constellations of decision-making in young people's educational trajectories». International Journal of Qualitative Studies in Education, 28 (3), 349-371.

$<$ https://doi.org/10.1080/09518398.2014.987850>. 